\title{
PROCESO Y FutURO dE LA INTEGRACIÓN DE CENTROAMÉRICA ${ }^{1}$
}

Jorge Antonio Giammattei Avilés

Presidente de la Corte Centroamericana de Justicia

I nicio mi intervención con la cita de un ilustre europeo universal que conoció mucho de integración puesto que siendo ciudadano romano, nacido en África, trasladado a Europa, es una persona que une a muchos, ya que sus ideas son compartidas por una gran mayoría de europeos y centroamericanos.

Me refiero a Agustín de Ipona, quién manifestó: "Gloriosa es la patria, estrecho el camino ... ¿̇porqué rehusa seguir el camino quien busca la patria?.

Traigo ante este Foro esa reflexión porque habiéndose suscrito todos los Tratados y Convenios necesarios para la integración de Centroamérica como Comunidad y no obstante las iniciativas para impulsarla y lograr una mayor integración regional como lo es este Diálogo, en los actuales momentos el proceso está, sino estancado, en una franca involución, por una crisis conceptual, axiológica y de responsabilidad en cuanto a lo que debe entenderse como integración regional comunitaria.

Creemos que la participación en un foro como el que hoy se realiza, es para expresarnos con toda franqueza sobre la forma de experimentar el Proceso y Futuro de la Integración de Centroamérica.

A ese respecto, sin mayores preámbulos y con el propósito de hacer el mejor uso del tiempo concedido a mi intervención, paso en forma extractada a exponer lo que el Tribunal que presido, con más de 9 años de existencia y por el hecho de ser considerado en el artículo 6 de su Convenio de Estatuto, "representante de la conciencia nacional de Centroamérica y depositaria y custodia de los valores que constituyen la nacionalidad centroamericana"; así como también por haber permanecido en forma continua desempeñando nuestras labores durante ese tiempo, nos permiten expresar con autoridad, algunos de los problemas, deformaciones y desnaturalización, existentes en el proceso de integración de Centroamérica.

${ }^{1}$ Exposición en el "Foro de Diálogo con la Sociedad Civil", celebrado en Bruselas, el 29 de enero del 2004, en el marco de la Cooperación Unión Europea y Centroamérica. 
Los Problemas, pueden ser reducidos a:

1. Desconocimiento de la naturaleza, objetivos, propósitos y principios de un proceso de Integración exitoso, como lo ha sido el europeo, por las máximas autoridades responsables del mismo.

2. Falta de una voluntad política, clara y definida, de parte de las autoridades mencionadas, por impulsar el proceso tal como está diseñado similar al europeo.

3. Falta de seriedad y responsabilidad de los Estados Miembros en el cumplimiento de los Tratados de Integración.

\section{a. Deformación}

La deformación se encuentra, sobre todo, de parte de las Autoridades de algunos Estados Miembros, que toman medidas unilaterales y dicen aceptar la integración "parcialmente", sólo en lo que consideran conveniente a sus intereses nacionales. Por ejemplo: uno, indicando que sólo acepta la integración en lo económico y no en otros campos. Otro, indicando que solo la acepta en lo político y no en lo demás. Uno más, que reduce el ingreso a una parte de su territorio a pobladores de los otros Estados, sin razón valedera. Igual se deforma por aquellos Estados Miembros que tratan de suscribir, separadamente, tratados de Libre Comercio con otras naciones o bloques. O bien, buscan un ingreso en otras comunidades.

\section{b. Desnaturalización}

Cuando se solicita a Organismos Internacionales y Bancos de la misma naturaleza (BID-CEPAL), la evaluación de un Sistema de origen regional, pasando por alto las facultades que a ese respecto tienen el PARLACEN y el Comité Ejecutivo del SICA.

Cuando la Reunión de Presidentes en Panamá II, confundiendo su función de Representantes de sus respectivos Estados, con la de Órgano Supremo del SICA, en contra de la naturaleza del Sistema (Acto contra natura), deciden en base a recomendaciones de Universidades y Organismos Financieros Internacionales, modificar la estructura y naturaleza misma del SICA, revirtiéndolo de un proceso de integración comunitaria a un proceso de colaboración intergubernamental en el que predomine únicamente el intercambio económico.

A tal grado es, que según lo ahí acordado y sin darle la debida participación a entes de la Comunidad, desnaturalizan y dejan inoperantes al PARLACEN y a la Corte Centroamericana de Justicia; y, al Comité Ejecutivo que representa los 
intereses de la Comunidad Centroamericana lo eliminan y lo sustituyen por un Comité de Enlace que representará los intereses de los Estados, ique desde luego ya lo estaban con los Consejos de Ministros!. En la misma forma eliminan a la Reunión o Foro de Vicepresidentes como de ninguna importancia o utilidad, no obstante su valioso trabajo en la Región del Trifinio y en la creación e instalación del PARLACEN.

Por si lo anterior no bastara en alguna Reunión de Presidentes, y en anteriores y posteriores acuerdos que han servido de base para implementar las reformas involutivas, no existió el consenso (unanimidad) que exige el artículo 14 del Protocolo de Tegucigalpa, en cuanto que uno de los Presidentes asistente, en algunas de ellas no firmó los acuerdos y en otros lo hizo como testigo.

Sobre lo observado con anterioridad, la Corte Centroamericana de Justicia ha hecho un llamado constante a las máximas autoridades responsables de la integración para que tomen acciones coherentes con sus discursos para impulsar la integración y además ha tomado iniciativas para fortalecer el proceso.

Entre las principales está la creación, el 28 de marzo de 1995, de la Comisión Permanente de Órganos Comunitarios Centroamericanos, integrada por representantes del Parlamento Centroamericano, la Secretaría General del SICA y de la misma Corte Centroamericana de Justicia, para, desde lo interno del Sistema SICA, revisar, evaluar y recomendar, periódicamente, sobre el estado de la Integración Centroamericana y el funcionamiento armónico de sus Órganos, Organismos e Instituciones, para transmitirlas por las instancias respectivas a la Reunión de Presidentes de Centroamérica, Órgano Supremo del Sistema, a efecto de que tengan conocimiento directo del sentir de los Órganos principales del referido Sistema. Actividad que durante los ya casi nueve años de existencia de la Comisión Permanente, hemos venido realizando sin que se haya atendido nuestras observaciones y recomendaciones por parte de la Reunión de Presidentes.

Esta Comisión Permanente, también ha acordado:

1. Exhortar al Consejo de Ministros de Educación del Sistema de la Integración Centroamericana incluir dentro de los respectivos programas de estudio de la educación primaria y secundaria la temática de la integración centroamericana como un objetivo indispensable en la formación de los estudiantes, que permita ir creando en las nuevas generaciones, una cultura de identidad regional, respecto de nuestro proceso de integración.

2. Encomendar a la Comisión de Educación, Cultura, Deporte, Ciencia y Tecnología del Parlamento Centroamericano, la elaboración de una propuesta para incluir el tema de la integración centroamericana dentro de un sis- 
tema educativo integral de los países del área, la cual será presentada a esta Comisión Permanente para su consideración y, en su caso, la aprobación de la misma y posterior presentación a las instancias respectivas del Sistema de la Integración Centroamericana (SICA), para que la incluya en sus programas. Esta propuesta deberá incluir los esfuerzos que actualmente realizan el Parlamento Centroamericano, la Corte Centroamericana de Justicia y la Secretaría General del SICA, respecto de esta temática, con organismos o instituciones que aporten lo necesario para los programas de post-grado y capacitación que implementen dichas instituciones.

3. Solicitar a la cooperación internacional, financiamiento para completar la restauración del edificio que alberga al "Centro de Altos Estudios de Derecho de Integración Dr. Roberto Ramírez", creado por la Corte Centroamericana de Justicia, con sede en la ciudad de Granada, Nicaragua, para que pueda servir como sede permanente para el desarrollo de programas formativos en materia de integración regional.

La Corte Centroamericana de Justicia en las Resoluciones Definitivas de las Consultas y Contenciosos que se han presentado, no se ha cansado de reiterar la importancia de los principios y propósitos que informan el Proceso de Integración de Centroamérica y que se encuentran expresamente detallados en los artículos 3 y 4 del Protocolo de Tegucigalpa a la Carta de la Organización de Estados Centroamericanos (ODECA), vigente para los ocho Estados Miembros: Belice, Costa Rica, El Salvador, Guatemala, Honduras, Nicaragua, República Dominicana y Panamá.

Propósitos y Principios que no solo forman el núcleo, el alma de la Comunidad a su mayor nivel normativo, como lo es estar contenidos en el Tratado Constitutivo de la misma, sino que de conformidad al artículo 9 del mismo: "Los Órganos e Instituciones del Sistema de la Integración Centroamericana, deberán guiarse por los propósitos y principios de este Protocolo e inspirarse en ellos tanto en sus decisiones, estudios y análisis como en la preparación de todas sus reuniones".

Con el mismo propósito, ha suscrito con 14 Instituciones Académicas, de Grado y de Post-grado, en toda Centroamérica, Convenios de Colaboración y Asistencia en la materia de integración comunitaria, celebrando numerosos seminarios y conferencias así como también elaborado folletos contentivos de lo que es y significa un proceso de integración para estudiantes de educación primaria y secundaria.

En la actualidad, tiene ya elaborado un Programa de Educación a Distancia de esta materia, que está para ser implementado desde su Centro de Altos Estudios de Derecho de Integración Dr. Roberto Ramírez. 
Como puede deducirse de lo expuesto, consideramos que es necesario tener una visión clara y común, por todos los Estados Miembros, de lo que es un Proceso de Integración exitoso, como el que lleva adelante la Unión Europea para que sirva de referencia y decidir, en forma responsable, la integración a realizarse en Centroamérica, que, por otra parte, es además querida por nuestros pueblos y necesaria para sobrevivir como unidad de destino en el proceso de globalización existente.

También consideramos que no obstante el contenido de los Tratados y Convenios suscritos y ratificados en esta materia, es necesario realizar una Conferencia Regional, precedida de las consultas que sean necesarias a los sectores oficiales y no oficiales de nuestras sociedades, para que participen, se involucren y sus opiniones sean consideradas en el proceso.

A ese respecto junto con el Parlamento Centroamericano (PARLACEN), la Corte Centroamericana está promoviendo una Gran Consulta de los Institutos Políticos, sectores organizados de la Sociedad Civil, Organismos de Gobierno y Órganos y Organismos e Instituciones regionales de integración, así como de la Sociedad Civil, con el propósito de determinar, qué tipo de integración es la que desean realizar, hasta dónde se desea profundizar, la naturaleza supranacional de las instituciones, en qué condiciones, en qué plazos, etc.

Sobre la base de lo acordado en esta Gran Consulta a los pueblos de Centroamérica, se convocaría a una Conferencia Regional sobre el futuro de la Integración de Centroamérica en la cual participarían los Estados Miembros y los Órganos Fundamentales de la Integración y así tomar las decisiones al más alto nivel político que nos conduzcan a nuevos y más profundos estadios de integración regional.

Creemos que es el mejor de los caminos, la mejor solución, el celebrar estas: Gran Consulta y Conferencia Regional, para determinar un proceso y futuro de la Integración de Centroamérica, con coherencia, responsabilidad y seguridad jurídica.

Sabemos que la Unión Europea ha tenido sus serias crisis y siempre las ha sabido superar, anteponiendo a los intereses propios de sus Estados Miembros, las de Europa. Está presente en nosotros, la Conferencia de Messina, de junio de 1955 y el relanzamiento europeo.

$\mathrm{Al}$ igual que en esa fecha, creemos que para Centroamérica es necesario también hoy: proseguir el establecimiento de una Centroamérica unida mediante el desarrollo de instituciones comunes, la fusión progresiva de las economías nacionales, la creación de un gran mercado común y la armonización progresiva de políticas sociales. 
Esperamos que las experiencias pasadas en la Unión Europea nos permitan, tomando su ejemplo, superar las nuestras.

Confiamos que en el marco de los objetivos y propósitos de este Foro y en el propósito de colaboración que anima a la Unión Europea hacia Centroamérica, se acuerde el apoyarnos técnica y financieramente en la celebración de la Gran Consulta y la Conferencsan ia Regional, y así definir el futuro de la Centroamérica Comunitaria.

Finalmente, debo referirme a lo relacionado con la Corte Centroamericana de Justicia y el "Programa de Apoyo a la Integración Centroamericana".

En primer lugar, debo manifestar en nombre de la Corte Centroamericana de Justicia, nuestro sentido agradecimiento por mejorar el papel y la contribución del Tribunal a la integración regional, a través del apoyo a sus reformas. Independientemente de nuestro acuerdo o no con las mismas, o con algunos aspectos de ellos, que deberían ser objeto de un amplio debate, estimamos que el primer paso que debe darse para ello, es que todos los Estados que participaron en la redacción, discusión, aprobación y suscripción del Convenio de Estatuto de la misma, lo ratifiquen; y, ya siendo Estados Parte de ese Convenio, se acuerde, por todos, en la forma ahí convenida y dándole participación al órgano supranacional ahí creado, las modificaciones pertinentes. Esto por elemental seguridad jurídica en el proceso de reforma a ese o cualquier otro Tratado o Convenio Multilateral.

En igual forma expresamos nuestro sentido agradecimiento por la contribución que se considera realizar a un centro de estudios relacionado con la integración, que, como ya lo hemos anticipado, está ya creado por la Corte Centroamericana y solicitada la colaboración para su establecimiento por la Comisión Permanente de los Órganos de la Integración Centroamericana.

También esperamos que todas aquellas Universidades e Instituciones Académicas con las que la Corte Centroamericana de Justicia tiene Convenios de Colaboración y que debido a ello y a las actividades conjuntas realizadas tienen avanzados conocimientos en la materia de Integración y Derecho Comunitario, puedan ser el núcleo o integrar parte de la red universitaria prevista. 